\title{
A Large Left Atrial Myxoma Obstructing the Mitral Valve Orifice
}

\author{
Md Nazmul Hasan \\ Dipal Krishna Adhikary \\ Tanjima Parvin
}

Department of Cardiology

University Cardiac Center

Bangabandhu Sheikh Mujib Medical University Dhaka, Bangladesh.

\section{*Correspondence to:}

\section{Dr. Md Nazmul Hasan}

Resident

Department of Cardiology

Bangabandhu Sheikh Mujib Medical University

Shahbag, Dhaka, Bangladesh.

Mobile: +8801675755638

E-mail: nazmulhasan45cmc@gmail.com

$w w w . c m o s h m c j . o r g$

\section{INTRODUCTION}

Cardiac tumors represent a relatively rare, yet challenging diagnosis. Secondary tumors are far more frequent than primary tumors of the heart. The majority of primary cardiac tumors are benign in origin, with primary malignant tumors accounting for $25 \%$ of cases. Left atrial myxoma is the most common intracardiac tumor. It could be seen in patients between 3-83 years of age, with the majority presenting in fifth decade of life as sporadic cases (90\%) and second decade as familial cases $(10 \%)^{1}$. It is an important source of central nervous system embolism². Elderly patients often present with nonspecific symptoms that are often overlooked in the absence of a supporting cardiac history which makes an early diagnosis challenging. This case report discusses an unusual presentation of a large left atrial myxoma in a 35 years old patient which almost completely obstructing the mitral valve orifice during diastole.

Key words : Atrial myxoma; Mitral valve; Palpitation.

\section{CASE REPORT}

A 35 years lady presented with a sixmonths history of shortness of breath and acute onset palpitations and chest pain. The chest pain confined mainly around left side, on heavy exertion and relieved on rest. It was progressive and presented even on light exertion like cooking and washing clothes and was associated with lightheadedness and chest discomfort even on doing daily activities since last two months. She was feeling fatigue which was progressive and was present even after doing daily activities for last two months. She also experienced palpitation for last six months which was progressive, precipitated by exertion, usually persisted for short duration and subsided spontaneously or by taking rest. Since last two months she experienced frequent episodes of palpitation even at rest. She denied any history of rheumatic fever in her childhood.She had been admitted three months ago with an episode of chest infection which was successfully treated with antibiotics.

On this occasion she was afebrile, mildly anemic, normotensive and had a regular pulse rate of $86 \mathrm{bpm}$. Precordial examination revealed slightly loud first heart sound with a localized mid-diastolic murmur, best heard at apical area in left lateral position with breath hold in expiration with the bell of stethoscope, without presystolic accentuation but variable with changes of position, increased during sitting and valsalva maneuvers. Further cardiovascular, respiratory, abdominal and neurological examination was unremarkable. A 12 lead electrocardiogram showed the features of left atrial and right ventricular hypertrophy with right bundle branch block. An in-patient trans-thoracic echocardiogram (TTE) demonstrated a mobile large left atrial mass $(2.8 \mathrm{~cm} \times 6.9 \mathrm{~cm})$ attached to the fossa ovalis region in the 
inter-atrial septum, prolapsing into the left ventricle through the mitral valve in diastole (Figures $1 \& 2$ ).

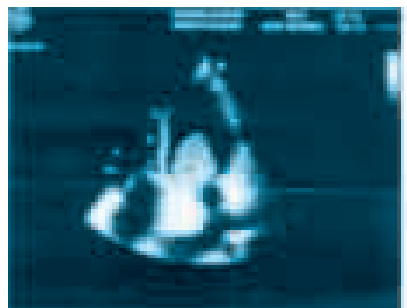

Figure 1 : Transthoracic echocardiographic (TTE) image of the left atrial myxoma. The figure shows the tumor in the left atrium (LA) in the apical four chamber view.

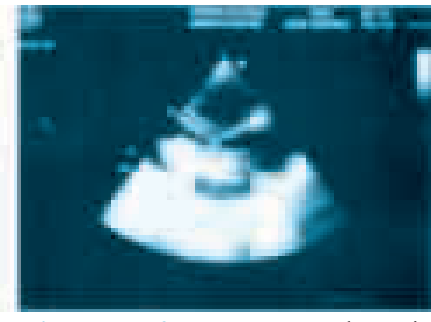

Figure 2 : Transthoracic echocardiographic (TTE) image of the left atrial myxoma obstructing the mitral valve. The Parasternal Long Axis view shows the tumor prolapsing through \& obstructing the mitral valve in diastole.

\section{DISCUSSION}

Cardiac tumors are a challenging and bizarre clinical situation. They are differentiated into primary and secondary (metastatic). The prevalence of primary cardiac tumors is $0.001-0.03 \%$ in autopsy series. Seventy-five per cent of primary tumors are benign in origin, with myxoma being the most frequent over $50 \%$ of cases. From the remaining $25 \%$ of malignant cardiac tumors, most frequent are cardiac sarcomas. Secondary tumors are from 20 - to 40 -fold more common than primary tumors, and $15 \%$ of patients suffering from any form of cancer exhibit metastases in the heart.

Atrial myxoma is the commonest $(20-30 \%$ of all) primary intra-cardiac tumour in adults and two thirds of these arise in the left atrium ${ }^{3}$. Other locations are right atrium (next commonest), ventricles, superior vena cava or pulmonary veins. In 5 percent of cases myxomas can be multiple. Differential diagnoses of atrial myxoma includes pedunculated thrombus, metastatic sarcoma and melanoma in left atrium ${ }^{4}$. Other metastatic tumors could specifically metastasize via inferior vena cava to the right side of the heart \& include hypernephroma, hepatoma, melanoma and intravenous leiomyomatosis from uterus.

Left atrial myxoma is most commonly seen in women with $90 \%$ being solitary and pedunculated and $10 \%$ being familial, with an autosomal dominant pattern of inheritance ${ }^{5}$. The mean age of onset is between $30-60$ years.
Most myxomas produce symptoms when they weigh greater than seventy grams. The presentation of atrial myxoma can in three different ways:

- Obstructive symptoms - dysponea, cardiac failure, dizziness, collapse \& syncope due to obstruction of the mitral valve.

- Constitutional symptoms - i.e. symptoms of autoimmune disease, vasculitis and various other non specific symptoms.

- Embolic symptoms - most frequently being cerebral emboli ${ }^{6}$.

TOE has nearly $100 \%$ sensitivity for cardiac myxoma. Atrial myxoma is usually seen at the border of the fossa ovalis in the left atrium, attached to the inter-atrial septum as in this case. The tumor tissue manifests as spherical/pedunculated mass attached to the endocardial surface with hypoechoic areas? TTE has less specificity than the TOE. Contrast CT demonstrates a well defined spherical or ovoid intracavitary mass. Magnetic resonance imaging (MRI) can visualise the point of attachment and helps differentiate a thrombus from a tumour. Differences in signal intensity between myocardium, tumor/thrombus is very helpful, especially with the use of contrast agent like Gadolinium-DTPA. A cine MRI sequence is a very sensitive technique to distinguish between an thrombus and a tumor, intra-cardiac or intravascular. Surgical excision is the only definitive treatment for atrial myxoma. In relatively small tumors, TTE/TOE can be used to monitor the growth of the tumor, to decide the timing of the surgery ${ }^{8}$. Conservative management is of limited value in symptomatic patients with large myxomas. However, a conservative strategy with TTE/TOE monitoring, \& anticoagulation is favoured in high operative risk patients, asymptomatic patients, and slow growing atrial myxomas.

The diagnosis of atrial myxoma can be elusive, especially when symptoms are suggestive of other diagnoses. In this case, the significance of this patient's past medical history of a transient ischemic attack only became apparent when the patient presented with new symptoms of AF, which led to various investigations looking for a source of cardiogenic cerebral embolism, eventually revealing the left atrial myxoma. This seems to be an unusual case due to the age at presentation of the patient. Left atrial myxoma presenting in seventh decade is rare, with only few published case reports, with this one of its first kind in the UK in the last ten years. Bire et alstudied the number of myxoma cases in patients over 75 years of age between 1962 and 1997 and found only 19 confirmed cases?.

\section{DISCLOSURE}

All the authors declared no competing interest.

\section{REFERENCES}

1. O'sRourke F, Dean N, Mouradian MS, Akhtar N, Shuaib A. A trial myxoma as a cause of stroke: case report and discussion. JAMC. 2003; 169:10.

2. Abascal VM, Kasznia J, Aldea G, Davidoff R. Left atrial myxoma and acute myocardial infarction: A dangerous duo in the thrombolytic era. Chest. 1996; 109:1106-1108.

3. Yap W, Bhattacharya K, Pathi V. Left atrial myxoma in transplanted heart. Heart J. 2005; 91:44-46.

4. Thakur AK, Thakur S. Infection \& cardiac tumors: Evaluation through Imaging. Medicine Update. Association of Physicians of India. 2003; 13:575-582.

5. Lamparter S, Moosdorf R, Maisch B. Giant left atrial mass in an asymptomatic patient. Heart J. 2004; 90:24-26.

6. Andrews R, Pollock G. Atrial Myxoma presenting as a pleural effusion and raised erythrocyte sedimentation rate of unknown cause. J R Soc Med. 1996; 89:585-586.

7. Engberding R, Daniel WG, Erbel R. The European Cooperative Study Group: Diagnosis of heart tumours by transoesophageal echocardiography: a multicentre study in 154 patients. Eur heart J. 1993; 14:1223-1238.

8. Bire F, Roudaut R, Chevalier JM, Quiniou G, Dubecq S, Marazanoff M, Choussat A. Cardiac myxoma in patients over 75 years of age. Am J Geriat Card. 2002; 11:165-168.

9. Ullah W, Mcgovern R. Natural history of an atrial myxoma. Age and ageing. 2005; 34:186-188. 